\title{
A New Adaptive Turbulence Model for Unsteady Flow Fields in Rotating Machinery
}

F. MAGAGNATO* and M. GABI

Department of Fluid Machinery, University of Karlsruhe, Kaiserstr. 12, D-76128 Karlsruhe, Germany

In order to predict unsteady flow fields one can use the Reynolds Averaged Navier-Stokes Equations (RANS) with a statistical turbulence model or Large Eddy Simulation (LES) in conjunction with a subgrid-scale model. Since the flow field in rotating machinery, internal combustion engines etc., is often strongly three-dimensional and unsteady, the calculation with LES and RANS are similar in terms of cpu-time providing the grid resolutions are similar.

The turbulence models used with RANS have been designed in order to capture all the turbulence effects since a steady state calculation cannot resolve any fluctuation. If one wants to perform an unsteady calculation, then a fraction of the turbulent fluctuations is already resolved by the numerical scheme, depending on the temporal and spatial resolution, and therefore the turbulence model must only model the unresolved part of the turbulence. The standard turbulence models used today cannot be used for such simulations, since they model always the whole turbulence spectrum.

On the other hand, the subgrid-scale models for LES model only a fraction of the turbulent spectrum, but they fail to model the turbulence in the limit of high cell Reynolds numbers (Speziale, 1998). A new adaptive turbulence model based on the popular two-equation models will be proposed which can be used for all cell Reynolds numbers in the unsteady case. It has the property that it reduces to a Direct Numerical Simulation (DNS) if the temporal and spatial resolution of the flow field is in the order of the Kolmogorov micro scale. If one does not resolve fluctuations (steady state) then the model reduces to a standard two-equation model. In between these two extremes it automatically adapts itself to the resolved turbulent fluctuations.

Keywords: CFD; Turbulence modeling; Unsteady flows; LES; Turbomachinery

Received 25 May 2000; in final form 7 July 2000.

* Corresponding author. Fax: 0049721608 3529. E-mail: magagnato@ mach.uni-karlsruhe.de
The flow in a turbomachine is strongly three-dimensional and unsteady. Nevertheless, the majority of the NavierStokes calculations conducted so far have been done as steady state computations due to the high computational effort needed for such a flow field. The inability of this approach to predict the pressure losses in accordance with the experimental observations has stimulated research in unsteady calculations (BRITE-EURAM, 1997). Another reason for an unsteady calculation is the prediction of the large scale vortices generated by thick trailing edges of turbine blades. It has been found that unsteady simulations predict the flow field in better agreement with experiments (Chen \& Leschziner, 1999; Magagnato, 1999) than steady state calculations. The turbulence models used where not modified in order to account for unsteady effects.

\section{BACKGROUND}

Turbulent flows are characterized by a wide range of length- and time-scales for Reynolds numbers of practical interest. The statistical approach uses the idea of a decomposition in mean values and fluctuations. Inserting this decomposition into the Navier-Stokes equations yields the averaged equations plus the unknown Reynolds stresses which have to be modeled. For the calculation of steady state solutions one usually solves the RANSequations together with a statistical turbulence model in order to model the total turbulence fluctuations which have been filtered out during the averaging process. For a DNS one needs to resolve all length- and time-scales, this means performing a time-dependent and three-dimensional calculation with very high temporal and spatial resolution (Piomelli, 1997). In between the statistical approach and the direct approach stands LES which is still threedimensional and time-dependent. Here the instantaneous flow field is also decomposed, but now into a resolved and an unresolved part with an characteristic space and time filter. LES still requires fairly fine meshes, but with the help 
of parallel computers it can be applied to more realistic flow fields of technological interest.

Traditionally LES has been done for fairly complex flow fields with a relatively high resolution of the turbulent scales and at relatively low Reynolds numbers. For these kind of computations, algebraic turbulence models (subgrid scale models) (Smagorinsky, 1963; Germano et al., 1991) suffice for modeling the unresolved turbulent effects since the large scales, which contain most of the energy, are numerically resolved, and the small scales are believed to be more universal in character so that they can be modeled by a simple isotropic model.

For the prediction of high Reynolds number flows in complex geometry's one cannot effort to resolve the flow field in the same way as in the simulations mentioned above, since than one had to solve a computational grid on the order of $10^{7}-10^{8}$ points with hundreds of thousands of time steps. This is not feasible in most technological applications, so one has to reduce the number of points at least one order of magnitude in order to be computable with a state-of-the-art supercomputer.

It is clear that with lower resolution the fraction of the turbulence spectrum which has to be modeled became larger, and so the contribution of the turbulence model becomes more important. It is believed by the authors that for such a coarse resolution a model based on transport equations for the turbulent kinetic energy and a turbulent timescale (or dissipation rate of the turbulent kinetic energy) is a better candidate than an algebraic model since it accounts also for the transport of the turbulence field.

\section{NEW TURBULENCE MODEL}

The idea for an adaptive turbulence model was motivated by the work of Speziale (1998) and Germano (1992). They suggested developing a combined methodology for the computation of complex turbulent flows. The filtering process of a chaotic flow field for the traditional Reynolds averaging and the implicit filter of the spatial filter in a LES are in principle different, but it was assumed that the implicit filtering process for LES becomes, in the limit of high cell Reynolds numbers, similar to the Reynolds averaging (Speziale, 1998).

An interesting new approach has been proposed by Spalart (Spalart et al., 1997). He estimates the computational costs of a high Reynolds number flow around an airfoil with a conventional LES. He concluded that such a calculation would be to expensive, so he proposed a Detached-Eddy Simulation (DES) which offers RANS in the boundary layers and LES after massive separation, within a single formulation. For this he modified the Spalart/Allmaras one equation model for the eddy viscosity (Spalart \& Allmaras, 1994) by replacing the length scale in their destruction term by the minimum between the local distance from the wall and the grid spacing. This model adapts automatically according to the spatial resolution.

An other approach will be proposed in this paper. In contrast to Spalart we base our approach on two-equation transport models, because we want to include a stochastic backscatter model based on the unresolved turbulent kinetic energy. Similar to Spalart we have tried to formulate a turbulence model which (1) converges asymptotically towards a statistical model in the limit of high cell Reynolds numbers, and, (2) in the other limit, approaches a direct numerical simulation when the length of the cell Reynolds number is on the order of the Kolmogorov length scale.

In order to achieve this, we propose to split the total turbulent kinetic energy $k$ and the total dissipation rate of the turbulent kinetic energy $\varepsilon$ (or the turbulent timescale $\tau$ ) into a resolved and an unresolved part.

$$
\begin{aligned}
& \varepsilon=\varepsilon^{\prime}+\bar{\varepsilon} \\
& k=k^{\prime}+\bar{k}
\end{aligned}
$$

The resolved turbulent kinetic energy must not be modeled since it is part of the solution, while the unresolved part must be modeled with a transport equation for $k^{\prime}$. In contrast, the resolved dissipation rate is not part of the solution and will be modeled by a relation for isotropic high Reynolds number flows. With this, the mean dissipation rate of the turbulent kinetic energy is proportional to the unresolved turbulent kinetic energy $k^{\prime}$ divided by the filter length scale $L_{\Delta}$ due to the numerical resolution,

$$
\bar{\varepsilon}=\frac{k^{13 / 2}}{L_{\Delta}}
$$

The unresolved dissipation rate will be calculated by a transport equation for $\varepsilon^{\prime}$. The same can be done with the resolved turbulent time scale $\tau^{\prime}$. Here the resolved turbulent time scale becomes

$$
\bar{\tau}=\frac{L_{\Delta}}{\sqrt{k^{\prime}}}
$$

Once we accept this, we are left with the determination of a suitable filter length scale. In many LES solutions the grid spacing is used as an implied filter width, although this may mask numerical errors. We choose also the grid spacing as an appropriate spatial length scale provided that the temporal filter is less or equal to the spatial resolution. Therefore we must choose the maximum filter length scale resulting from the spatial and the temporal filter width. For the spatial filter we use the common filter width

$$
L_{s}=2 \cdot \sqrt[3]{\Delta x \cdot \Delta y \cdot \Delta z}
$$


where $\Delta x, \Delta y$ and $\Delta z$ is the cell size in the different coordinate direction, while for the temporal filter we use

$$
L_{t}=|u| \cdot \Delta t
$$

for each cell, with $|u|$ being the velocity in the node and $\Delta t$ is the time step. Taking the maximum results in

$$
L_{\Delta}=\max \left\{\begin{array}{l}
L_{s} \\
L_{t}
\end{array}\right\}
$$

This enables us to introduce this splitting into any turbulence model that contains a transport equation for $k$ and $\varepsilon$ or $\tau$. We choose a slightly modified nonlinear (cubic) two-equation model proposed by $\mathrm{Craft} /$ Launder/Suga (1995) but transform it mathematically into an equivalent $k-\tau$-formulation for numerical reasons (Magagnato, 1999). This model gives reasonable results for a wide range of flow fields including turbomachinery flow. It reads:

$$
\begin{aligned}
& \frac{\partial\left(\rho k^{\prime}\right)}{\partial t}+\frac{\partial\left(\rho u_{i} k^{\prime}\right)}{\partial x_{i}}=\frac{\partial}{\partial x_{i}}\left(\left(\mu+\mu_{k}\right) \frac{\partial k^{\prime}}{\partial x_{i}}\right) \\
& +P_{k}-\rho \frac{k^{\prime}\left(1+\frac{\tau^{\prime} \sqrt{k^{\prime}}}{L_{\Delta}}\right)}{\tau^{\prime}} \\
& \frac{\partial\left(\rho \tau^{\prime}\right)}{\partial t}+\frac{\partial\left(\rho u_{i} \tau^{\prime}\right)}{\partial x_{i}}=\frac{\partial}{\partial x_{i}}\left(\left(\mu+\mu_{\tau}\right) \frac{\partial \tau^{\prime}}{\partial x_{i}}\right) \\
& +\left(1-c_{\varepsilon_{1}}\right) \cdot \frac{\tau^{\prime}}{k^{\prime}} P_{k} \\
& +\rho\left(c_{\varepsilon_{2}}-\left(1+\frac{\tau^{\prime} \sqrt{k^{\prime}}}{L_{\Delta}}\right)\right) \\
& +\frac{2}{k^{\prime}}\left(\mu+\mu_{\tau}\right) \frac{\partial k^{\prime}}{\partial x_{i}} \frac{\partial \tau^{\prime}}{\partial x_{i}} \\
& -\frac{2}{\tau^{\prime}}\left(\mu+\mu_{\tau}\right) \frac{\partial \tau^{\prime}}{\partial x_{i}} \frac{\partial \tau^{\prime}}{\partial x_{i}} \\
& -0.06 \cdot S \cdot \tau^{\prime^{3}}\left(\frac{\partial^{2} u_{i}}{\partial x_{k} \partial x_{j}}\right)^{2} \\
& \mu_{t}=\rho \cdot c_{\mu} \cdot f_{\mu} \cdot k^{\prime} \cdot \tau^{\prime} \quad c_{\mu}=\frac{0.3}{1+0.35 \cdot S^{3 / 2}} \\
& \left(1-\exp \left(-\frac{0.36}{\exp (-0.75 \cdot S)}\right)\right) \\
& P_{k}=-\rho \overline{u_{i}^{\prime} u_{j}^{\prime}} \frac{\partial u_{i}}{\partial x_{j}} \quad S=\tau^{\prime} \sqrt{S_{i j} S_{i j} / 2} \\
& S_{i j}=\frac{\partial u_{i}}{\partial x_{j}}+\frac{\partial u_{j}}{\partial x_{i}}-\frac{2}{3} \frac{\partial u_{i}}{\partial x_{i}} \delta_{i j} \\
& \Omega_{i j}=\frac{\partial u_{i}}{\partial x_{j}}-\frac{\partial u_{j}}{\partial x_{i}} \\
& f_{\mu}=1-\exp \left(-\left(\frac{\mathrm{Re}_{t}}{90}\right)^{1 / 2}-\left(\frac{\mathrm{Re}_{t}}{400}\right)^{2}\right)
\end{aligned}
$$

$$
\begin{aligned}
-\rho \overline{u_{i}^{\prime} u_{j}^{\prime}}= & \mu_{t} \cdot S_{i j}-\frac{2}{3} k^{\prime} \delta_{i j}-c_{1} \mu_{t} \tau^{\prime} \\
& \left(S_{i k} S_{k j}-\frac{1}{3} S_{k l} S_{k l} \delta_{i j}\right) \\
& -c_{2} \mu_{t} \tau^{\prime}\left(\Omega_{l k} S_{k j}+\Omega_{j k} S_{k j}\right) \\
& -c_{3} \mu_{t} \tau^{\prime}\left(\Omega_{l k} \Omega_{j k}-\frac{1}{3} \Omega_{k l} \Omega_{k l} \delta_{i j}\right)+c_{\mu} \mu_{t} \tau^{\prime 2} \\
& \left\{c_{4}\left[S_{k l} \Omega_{l j}+S_{k j} \Omega_{l i}-\frac{2}{3} S_{k m} \Omega_{l m} \delta_{i j}\right]\right. \\
& S_{k l}+c_{5}\left[S_{i k} S_{j l}-\frac{1}{3} S_{m k} S_{m l} \delta_{i j}\right] S_{k l} \\
& \left.\left.+c_{6} S_{i j} S_{k l} S_{k l}+c_{7} S_{i j} \Omega_{k l} \Omega_{k l}\right\}\right\}
\end{aligned}
$$

With the constants (see Table I).

The boundary conditions for this model must also reflect the combined methodology mentioned above in that they must reduce to the standard boundary conditions used for the statistical model and to the specification of fluctuations in the limit of a direct numerical simulation. This will be accomplished with the following approximation.

The resolved part of the turbulent kinetic energy is applied in the simulation as disturbances of the freestream velocity field with a random white noise while the unresolved part is used as the boundary condition of the transport equation of $k^{\prime}$. The splitting is done assuming that the energy spectrum of the turbulent kinetic energy in the freestream is represented by the Kolmogorov law proportional to $k^{-5 / 3}$. Then the splitting will be done between the two limits of the wavenumber of the Kolmogorov microscale $\kappa_{\eta}=\pi / L_{\eta}$ and the wavenumber of the characteristicscale $\kappa_{L}=\pi / L_{T}$, built with the usual characteristic turbulent length scale used for a conventional two-equation model. The split point is given by the wavenumber of the grid scale $\kappa_{\Delta}=\pi / L_{\Delta}$, were $L_{\Delta}$ is given above.

Now the splitting will be done by integrating the spectrum of the turbulent kinetic energy between $k_{L}$ and $k_{\eta}$ with a linear weighting of the split point $\kappa_{\Delta}$ (see Figure 1). This results in:

$$
k^{\prime}=k_{\infty} \frac{\kappa_{\eta}^{-2 / 3}-\kappa_{\Delta}^{-2 / 3}}{\kappa_{\eta}^{-2 / 3}-\kappa_{L}^{-2 / 3}}
$$

where the wavenumber of the Kolmogorov micro-scale is approximated by:

$$
\kappa_{\eta}=\kappa_{L}\left(\frac{\rho_{\infty} L_{T} \sqrt{k_{\infty}}}{\mu_{\infty}}\right)^{-3 / 4} .
$$

TABLE I Constants of the adaptive model

\begin{tabular}{cccccccc}
\hline $\mathrm{C}_{\mu}$ & $\mathrm{C}_{1}$ & $\mathrm{C}_{2}$ & $\mathrm{C}_{3}$ & $\mathrm{C}_{4}$ & $\mathrm{C}_{5}$ & $\mathrm{C}_{6}$ & $\mathrm{C}_{7}$ \\
\hline 0.09 & -0.1 & 0.1 & 0.26 & -0.081 & 0 & 0.0405 & -0.0405 \\
\hline
\end{tabular}




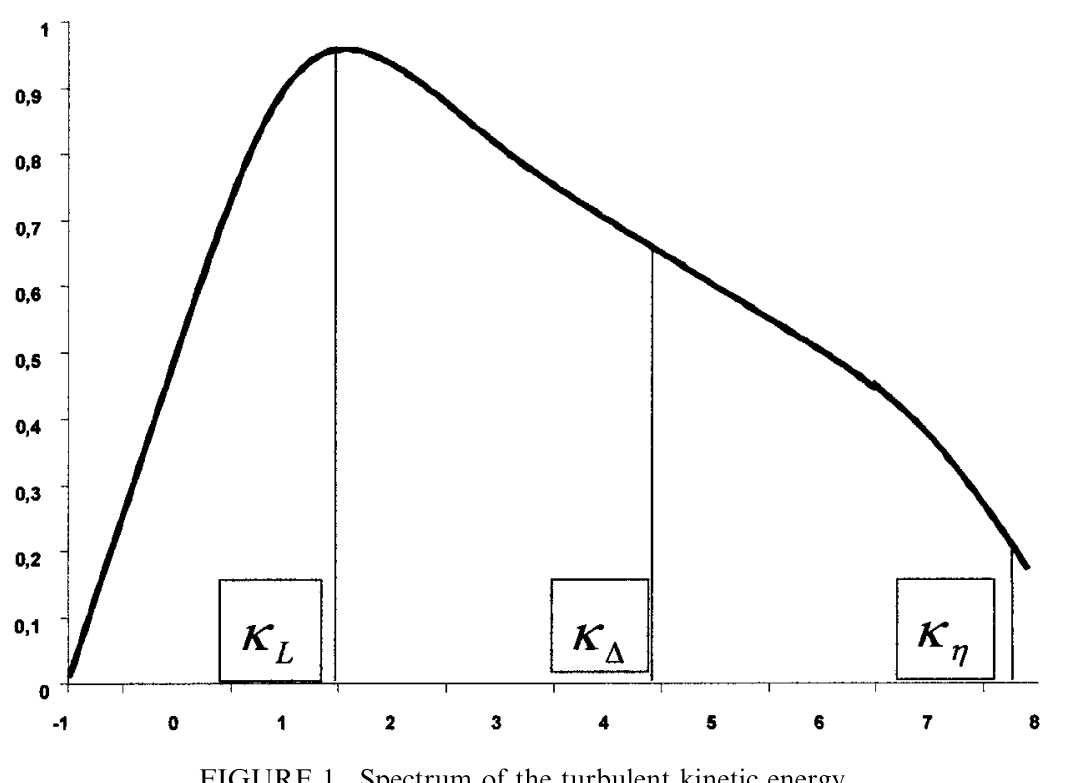

The boundary condition for $\tau^{\prime}$ will be given by

$$
\tau^{\prime}=\frac{L_{\Delta}}{\sqrt{k_{\infty}}} .
$$

With this boundary condition the limits $k^{\prime}=k_{\infty}$ for $\kappa_{\Delta}=\kappa_{L}$ and $k^{\prime}=0$ for $\kappa_{\Delta}=\kappa_{\eta}$ are recovered.

It is well known (Schumann, 1995) that the energy exchange between large scale motion and small scale motion is not unidirectional. Energy is exchanged in both directions, but in the mean it cascades towards the small scale motion until it is dissipated by the viscous process. The exchange process from the large scales to the small scales is called forward scatter while the opposite direction is called backscatter. The forward scatter is dissipative in nature and is usually modeled with an eddy viscosity model, for example Smagorinsky-Lilley (Lilley, 1967) or the above mentioned model. In contrast, the backscatter is stochastic in nature. There were only a few attempts to use a backscatter model in the past (Leith, 1990), (Mason and Thomson, 1992), (Schumann, 1995), but it appears to be very important to include such a model into a LES. Schumann proposed a backscatter model which gives good results for decaying isotropic turbulence. We use his model as a basis to derive a backscatter model based on the unresolved turbulent kinetic energy $k^{\prime}$ and the unresolved turbulent timescale $\tau^{\prime}$ from our new model which should have the correct asymptotical behavior. This means in the limit of a DNS its velocity disturbances should disappear and in the limit of a RANS they should become constant. In between it should have a monotonic behavior with a maximum in the inertial subrange. This backscatter model reads:

$$
-\left(\rho \overline{u_{i}^{\prime} u_{j}^{\prime}}\right)_{B}=-\rho v_{i}^{\prime} v_{j}^{\prime}+\frac{2}{3} \rho k^{\prime} \delta_{i j}
$$

The random velocities $v_{i}^{\prime}$ are then calculated at each time step using a Langevin-type equation,

$$
\begin{aligned}
\left(v_{i}^{\prime}\right)^{n}= & \left(v_{i}^{\prime}\right)^{n-1}\left(1-\frac{\Delta t}{\tau^{\prime}}\right) \\
& +\left(\frac{\Delta t}{\tau^{\prime}}\left(2-\frac{\Delta t}{\tau^{\prime}}\right) \frac{2}{3} k^{\prime}\right)^{1 / 2} Z_{i}^{n}
\end{aligned}
$$

were $k^{\prime}$ is the unresolved turbulent kinetic energy and $Z_{i}$ is an independent random number in the range $(-1,1)$. With the initialization at the first time step the random disturbance becomes:

$$
\left(v_{i}^{\prime}\right)^{0}=\left(\frac{2}{3} k^{\prime}\right)^{1 / 2},
$$

and

$$
\left\langle\left(\left(v_{i}^{\prime}\right)^{n}\right)^{2}\right\rangle=\frac{2}{3} k^{\prime} .
$$

Here $\Delta t$ is again the time step of the numerical scheme and $\tau^{\prime}$ as defined in Eq. [11]. One can immediately recognize that this model gives the correct asymptotical results. In the limit of a DNS $k^{\prime}=0$ and $v_{i}^{\prime}=0$. On the other side if $L_{\Delta}=\infty$ then $\tau^{\prime}=\infty$ and $\left(v_{i}^{\prime}\right)^{n}=\left(v_{i}^{\prime}\right)^{0}$ for all time steps and the stochastic stresses of the backscatter model are zero.

The complete model for the Reynolds stresses or subgrid-scale stresses now are applied in the following way. The Reynolds (subgrid-scale) stresses for a $k-\tau$ model are calculated according to:

$$
-\rho \overline{u_{i}^{\prime} u_{j}^{\prime}}=\rho c_{\mu} k^{\prime} \tau^{\prime} S_{i j}-\frac{2}{3} \rho k^{\prime} \delta_{i j}-\rho v_{i}^{\prime} v_{j}^{\prime}+\frac{2}{3} \rho k^{\prime} \delta_{i j}
$$


For a $k-\varepsilon$ model:

$$
-\rho \overline{u_{i}^{\prime} u_{j}^{\prime}}=\rho c_{\mu} \frac{k^{\prime 2}}{\varepsilon^{\prime}} S_{i j}-\frac{2}{3} \rho k^{\prime} \delta_{i j}-\rho v_{i}^{\prime} v_{j}^{\prime}+\frac{2}{3} \rho k^{\prime} \delta_{i j}
$$

\section{NUMERICAL METHOD}

The numerical method used is a block structured finite volume cell centered scheme (Magagnato, 1998). A 4th order Runge-Kutta scheme is used for integration in time, while in space a 4th order cell centered scheme has been used. A number of different turbulence models are available ranging from simple algebraic models to non-linear two equation models. The compressible Navier-Stokes equations can be solved as a RANS, LES or a DNS. The additional computational time for the new model is negligible $(1-2 \%)$ compared to a conventional two-equation turbulence model if one calculates the filter length $L_{s}$ once at the initialization and store it into an extra permanent array. The code is very carefully parallelized with message passing interface (mpi) in order to speed up the computation efficiently. Calculations have been made mostly on a IBM SP2 using 64 processors in parallel (efficiency 93\%). For more details please refer to Magagnato, 1998.

\section{RESULTS}

Two flow fields have been investigated in order to study the new turbulence model and compared with the standard turbulence models. The first test case is the flow around a circular cylinder. Measurements have been conducted by Cantwell and Coles (1983).

The Reynolds number of this flow field is $\mathrm{Re}=140,000$ based on the diameter of the cylinder and the freestream velocity of $u=21.2 \mathrm{~m} / \mathrm{s}$. The turbulence level is $T u=0.6 \%$. Since the characteristic turbulence length scale in the freestream is not known, the common practice is to choose the turbulence length scale such that the resulting eddy viscosity in the freestream is on the order of the laminar viscosity. The turbulence level then is used for the specification of the total turbulent kinetic energy and the turbulent length scale in the freestream for the statistical models. For the LES with the adaptive model the above mentioned boundary conditions have been used.

An O-H-type grid has been generated with a total of approximately 18,000 points in the symmetry plane and 97 points in the spanwise direction (see Figure 2), giving a total of approximately $1,700,000$ points. In the three dimensional computation the cylinder has been extended three diameters in the spanwise direction in order to resolve the crossflow correlations sufficiently.

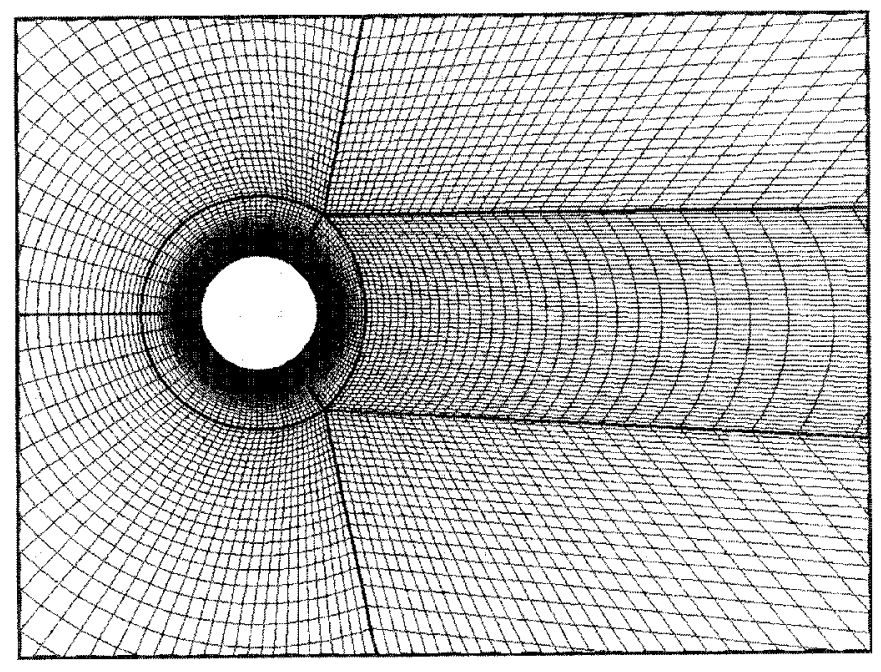

FIGURE 2 Computational grid of the circular cylinder.

Two unsteady and two-dimensional calculations have been made with conventional two-equation models. In order to demonstrate mesh independents a sequence of coarser grids are solved. The coarse grid consisting of about 1000 points showed no vortex shedding due to the numerical damping of the fluctuations. In the middle grid (about 4500 points) the flow field started to shed vortices, also they were numerically damped after about four diameters downstream of the cylinder. The mean pressure distribution was almost equal to that obtained in the finest grid indicating a mesh converged solution.

The comparison of the measured mean pressure distribution with the results obtained with the linear model of Launder/Sharma as well as the non-linear model of Craft/Launder/Suga shows poor agreement (see Figure 3). The reason is that the strongly three-dimensional vortices in the wake of the cylinder are not resolved and hence the strong non-linear vortex interaction is suppressed.

The LES with the adaptive model predicts a threedimensional flow field. In Figure 4 the three-dimensionality of the flow field is visualized by showing the crossflow components in the wake (the blue isosurface corresponds to $w=30 \mathrm{~m} / \mathrm{s}$ while the red isosurface to $w=30 \mathrm{~m} / \mathrm{s}$ ). The mean pressure distribution predicted by the adaptive model is compared with the observations of Cantwell/Coles in Figure 5. The agreement with the measurements is greatly improved.

Similar to the two dimensional case the flow field in the coarse grid (about 28,000 points) showed no vortex shedding and the maximum value of the ratio between the eddy viscosity and the molecular viscosity was approximately $v_{t}=130$. In the middle grid (about 210,000 points) the vortex generation process starts to develop but the flow field remained essentially two dimensional. The maximum value of the eddy viscosity ratio was in the order of $v_{t}=50$. 


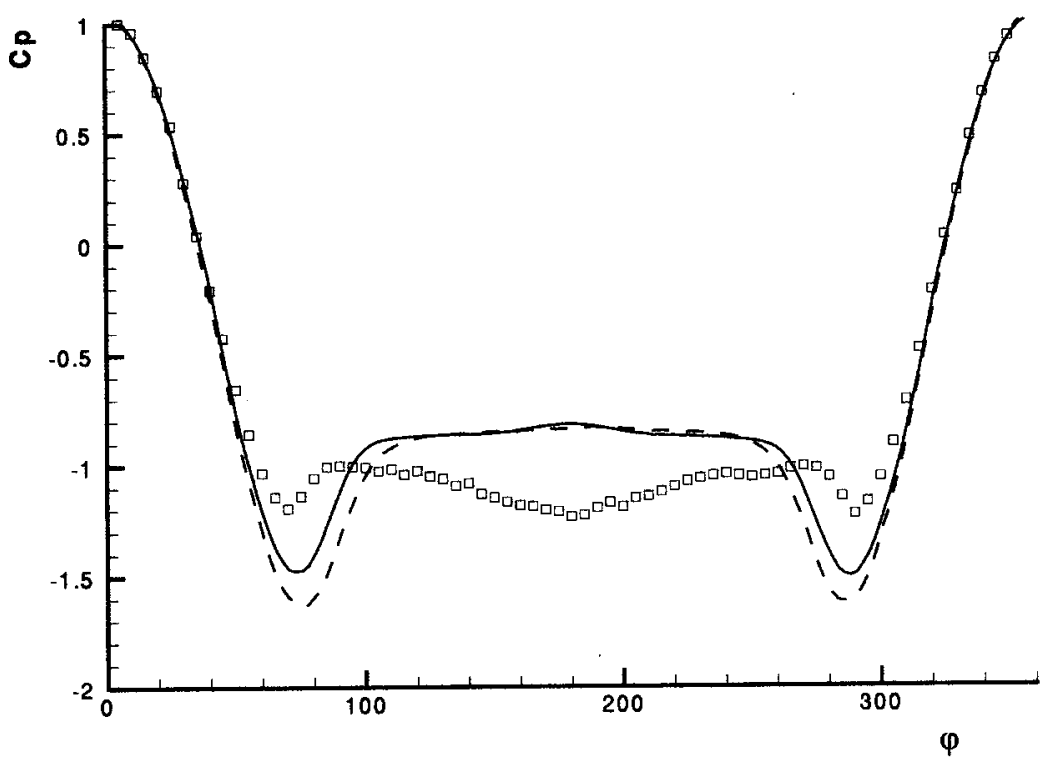

FIGURE 3 Mean pressure distribution on the circular cylinder calculated with ---- Speziale et al., model and - Craft/Launder/Suga model $\square \square \square$ experiment.

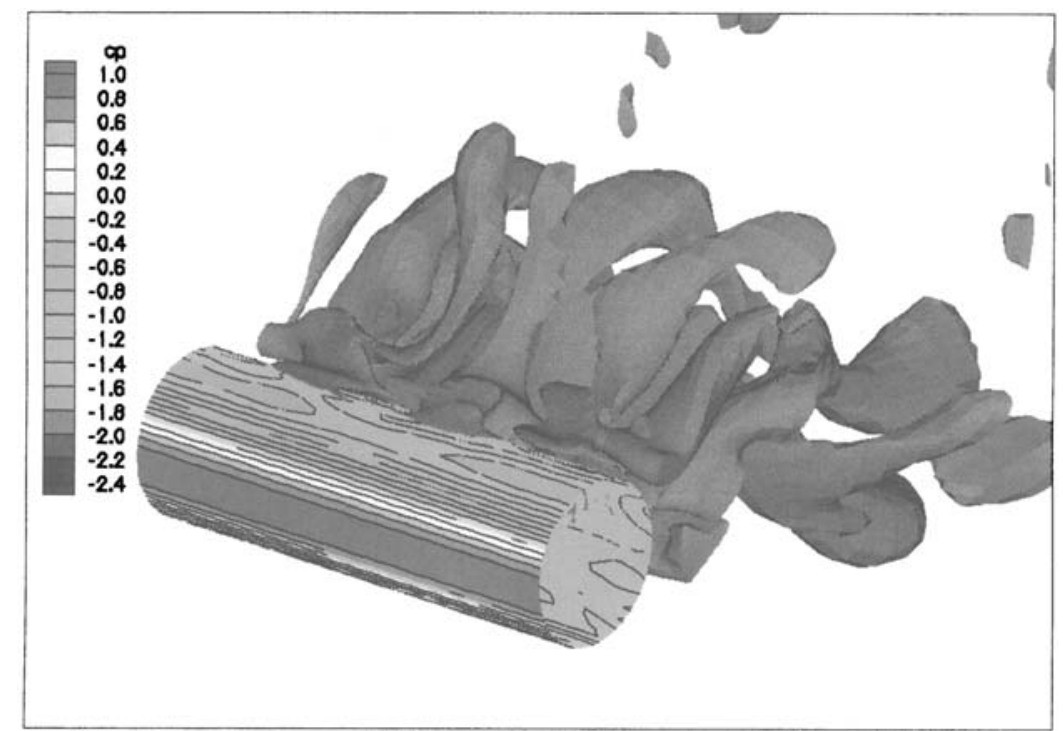

FIGURE 4 Crossflow velocity isosurface in the wake of the circular cylinder calculated with the adaptive model (See Colour Plate at back of issue.).

On the finest grid the maximum eddy viscosity ratio dropped below $v_{t}=20$. The excessive damping of the flow instabilities was reduced and a three dimensional flow field established.

The new adaptive model has also been applied to the unsteady flow field past the VKI-turbine blade (see Figure 6) with a thick trailing edge and compared with standard two-equation models. Again the flow was calculated to a linear, a nonlinear and the adaptive model. Measurements have been conducted by Ubaldi et al. (1996). The Reynolds number $\operatorname{Re}_{2 \text { is }}=1.6 \cdot 10^{6}$ is based on the chord length $s=0.3 \mathrm{~m}$ and the isentropic exit Mach number $\mathrm{Ma}_{2 \mathrm{is}}=0.23$. The measured turbulence level was $T u=3 \%$. No turbulence length scale has been measured. The decay of the turbulent kinetic energy along the turbine blade has been measured. In order to match these values, a turbulence length scale of $L_{t}=0.018 \mathrm{~m}$ was applied at the upstream boundary. The grid has been generated for this test case with about 72,000 points for a two dimensional calculation. Only one blade has been calculated using periodic boundary conditions to simulate the other blades. The calculations have been done using 


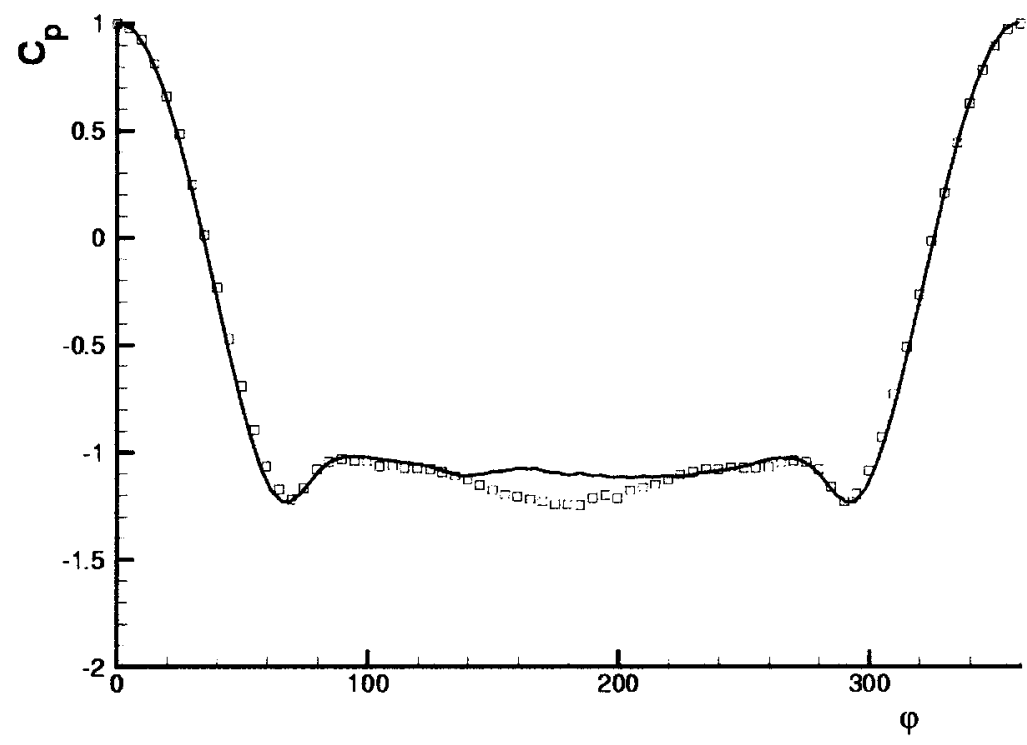

FIGURE 5 Mean pressure distribution on circular cylinder calculated with the — adaptive model $\square \square \square$ experiment.

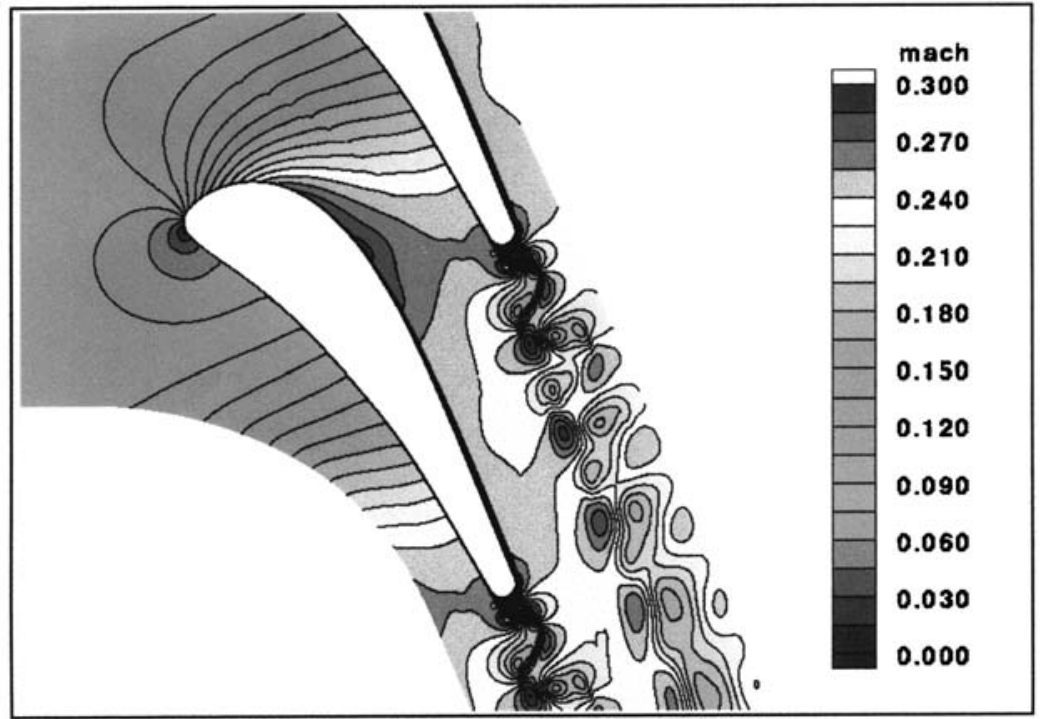

FIGURE 6 Mach number distribution for the VKI turbine blade with vortex shedding at the trailing edge (See Colour Plate at back of issue.).

a 2nd order as well as a 4th order cell centered scheme.

Again a mesh convergence study has been made with a total of four different grids $(1,100,4,500,18,000$ and 72,000 points). Due to the greater mesh resolution the vortex shedding process started on the second coarsest grid $(4,500$ points) but with a very small extend in the near wake of the trailing edge. The solution on the middle grid showed again almost the same results as with the fine grid. The comparison of the mean pressure distribution between the middle grid and the fine grid was practically undistinguishing from each other.
A vortex shedding frequency of $f=1650 \mathrm{~Hz}$ has been calculated with the new model using the 4th order scheme (see Figure 7). It is very close to the experimental value (Ubaldi et al., 1996) of $f=1700 \mathrm{~Hz}$ while the same model gives $f=1560 \mathrm{~Hz}$ using the $2 \mathrm{nd}$ order scheme. The nonlinear two-equation model proposed from Craft/Launder/ Suga on the other hand predicts the vortex shedding frequency of about $f=1180 \mathrm{~Hz}$ with the 4 th order scheme while it gives $f=1080 \mathrm{~Hz}$ with the 2 nd order scheme. The linear two-equation model of Launder/Sharma fails to predict the vortex shedding completely with both numerical schemes due to the very high eddy viscosity predicted 


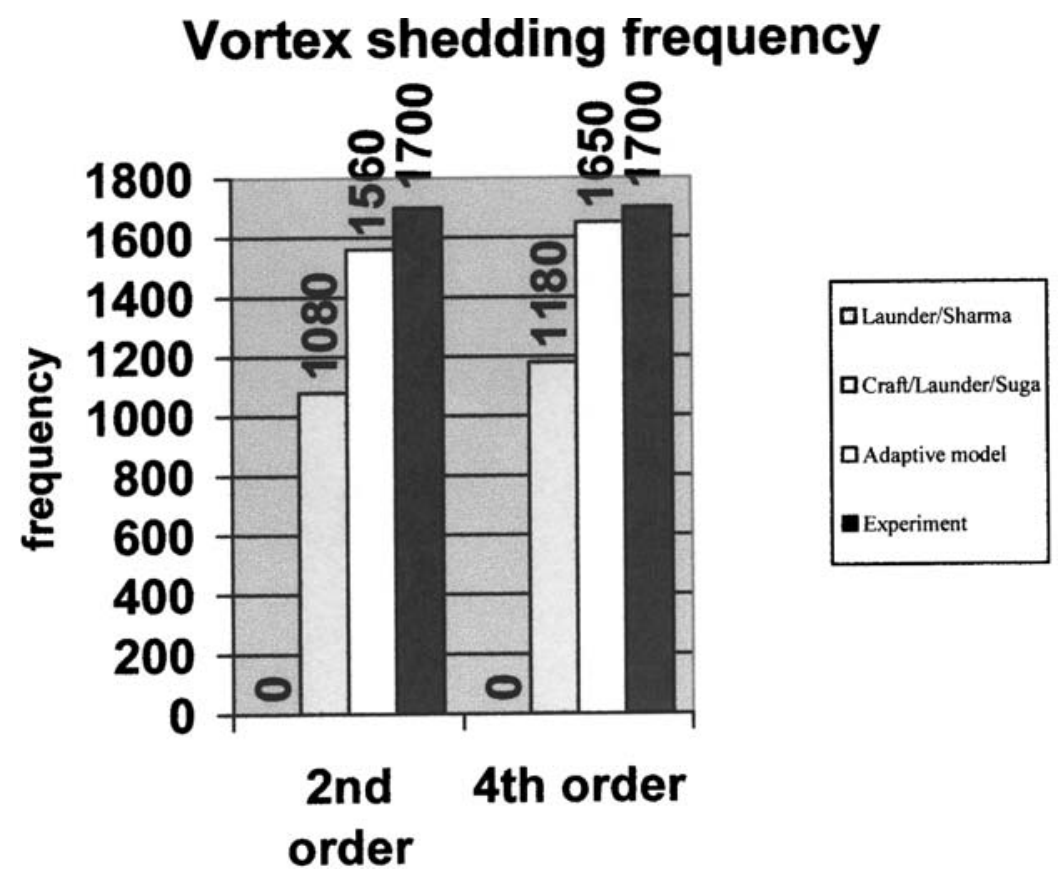

FIGURE 7 Vortex shedding frequency of the turbine blade.

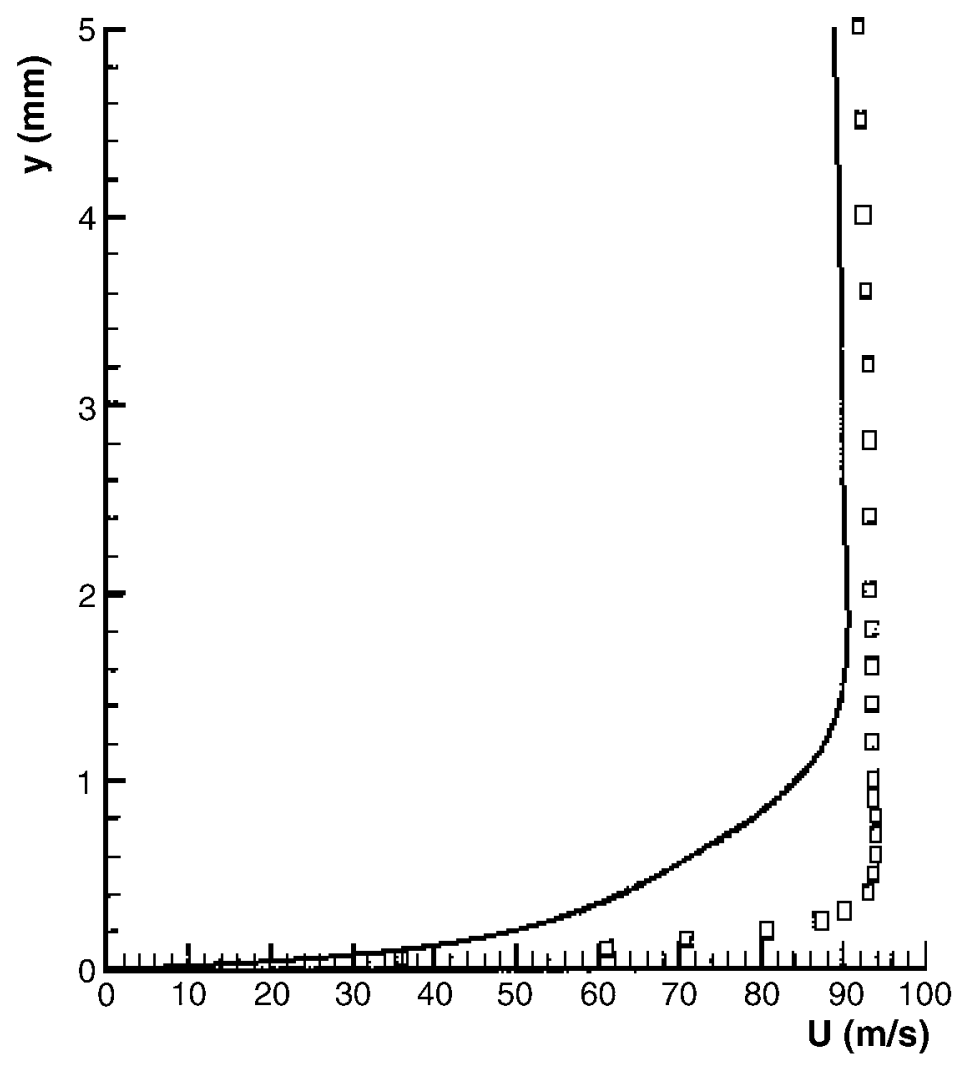

FIGURE 8 Mean velocity profile calculated with the linear two-equation model at $30 \%$ chord on the suction side of the turbine blade. 


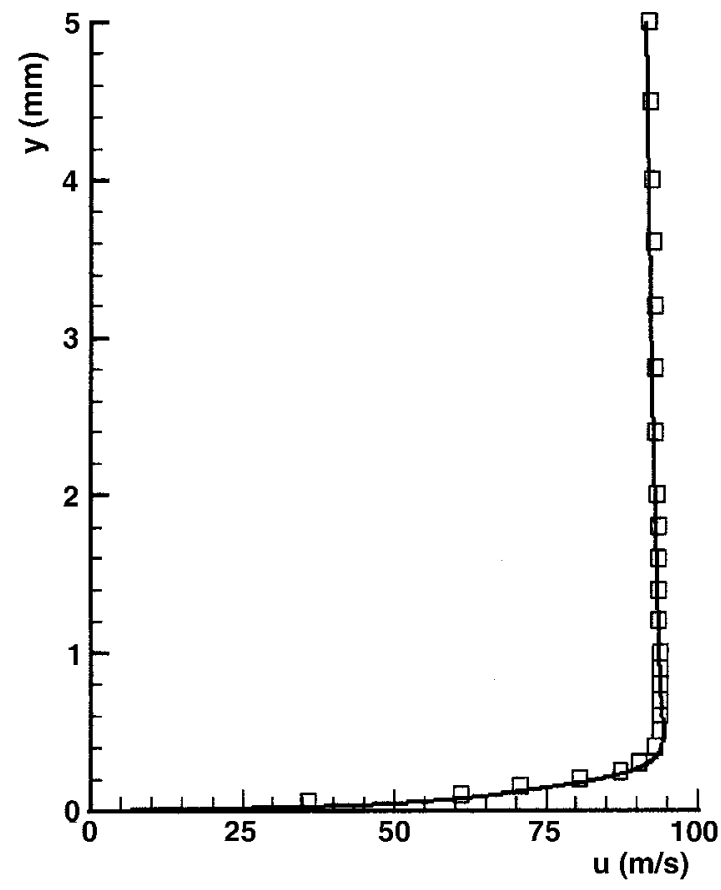

FIGURE 9 Mean velocity profile calculated with the adaptive two-equation model at $30 \%$ chord on the suction side of the turbine blade.

with these model suppressing completely the vortex shedding mechanism.

The mean pressure distribution is not very sensitive in this case. The results obtained with the different turbulence models were very close to each other and in good agreement with the experiment. The velocity profiles calculated with the new model are in good agreement with the experiment (see Figure 8) while the linear two-equation models disagree with the experimental profiles (see Figure 9) because the eddy viscosity in the boundary layer and hence the turbulent stresses are too high.

\section{CONCLUSION}

A new procedure has been proposed, which leads to an adaptive turbulence model for unsteady flow fields. It gives the correct asymptotical limit of a direct numerical simulation, if all turbulent fluctuations are resolved and reduce to a conventional turbulence model, if no fluctuations at all are resolved. This procedure can be applied to any two equation turbulence model which solves a transport equation for the turbulent kinetic energy $k$ and either the dissipation rate of the turbulent kinetic energy $\varepsilon$ the turbulent time scale $\tau$ or the inverse time scale $\omega$. A stochastic backscatter model is also part of the new procedure with the same asymptotical behavior as mentioned above.

The procedure has been used to derive a non-linear $k-\tau$ model which predicts the flow field around a circular cylinder and the flow around a turbine blade in good agreement with the experiments. The additional computational overhead for this new model is very low.

\section{REFERENCES}

Cantwell, B. and Coles, D. (1983) An Experimental Study of Entrainment and Transport in the Turbulent near Wake of a Circular Cylinder, Journal of Fluid Mechanics, pp. 321-374.

Chen, W. L. and Leschziner, M. (1999) Turbulence modelling of rotorstator interaction with linear and non-linear eddy-viscosity models, Third European Conference on Turbomachinery: Fluid Dynamics and Thermodynamics, A, 259-268.

Craft, T. J., Launder, B. B., and Suga, K. (1995) A Non-linear Eddy Viscosity Model including Sensitivity to Stress Anisotropy, 10th Symposium Turbulent Shear Flows, Pennsylvania State University.

Germano, M. (1992) Turbulence: the filtering approach, J. Fluid Mech., 238, $325-336$.

Leith, C. E. (1990) Stochastic backscatter in a subgrid-scale model: plane shear mixing layer, Phys. Fluids A, 2, 297-299.

Lilly, D. K. (1967) The representation of small-scale turbulence in numerical simulation experiments, Proc. IBM Sci. Comput. Symp. On Environmental Sci., pp. 195-210.

Mason, P. J. and Thomson, D. J. (1992) Stochastic backscatter in large eddy simulations of boundary layers, Journal of Fluid Mechanics, 242, $51-78$.

Magagnato, F. (1998) 'KAPPA' -Karlsruhe Parallel Program for Aerodynamics, TASK Quarterly, 2(2), 215-270.

Magagnato, F. (1999) Unsteady flow past a turbine blade using non-linear two-equation turbulence models, Third European Conference on Turbomachinery: Fluid Dynamics and Thermodynamics, A, 221-230.

Piomelli, U. (1997) Introduction to the modelling of turbulence, von Karman Institute for Fluid Dynamics, Lecture Series 1997-03.

Schumann, U. (1995) Stochastic backscatter of turbulence energy and scalar variance by random subgrid-scale fluxes, Proc. R. Soc. London A, 451, $293-318$.

Smagorinsky, J. (1963) General circulation experiments with the primitive equations. I. The basic experiment, Mon. Weather Review 91, pp. $91-164$.

Spalart, P. R. and Allmaras, S. R. (1994) A one-equation turbulence model for areodynamic flows. La Recherche Aerospatiale, 1, 5-21.

Spalart, P. R., Jou, W.-H., Strelets, M., and Allmaras, S. R. (1997) Comments on the feasibility of LES for wings, and on a hybrid RANS/ LES Approach, First AFORS Intern. Conference on Direct Numerical Simulation and Large Eddy Simulation.

Speziale, C. G., Abid, R., and Anderson, E. C. (1990) A Critical Evaluation of Two-Equation Models for Near Wall Turbulence, ICASE Report No. 90-46.

Speziale, C. G. (1998) Turbulence Modeling for Time-Dependent RANS and VLES: A Review, AIAA Journal, 36(2), 173-184.

Ubaldi, M., Zunino, P., Campora, U., and Chiglione, A. (1996) Detailed Velocity and Turbulence Measurements of the Profile Boundary Layers in a Large Scale Turbine Cascade, ASME-Journal 96-GT-42. 

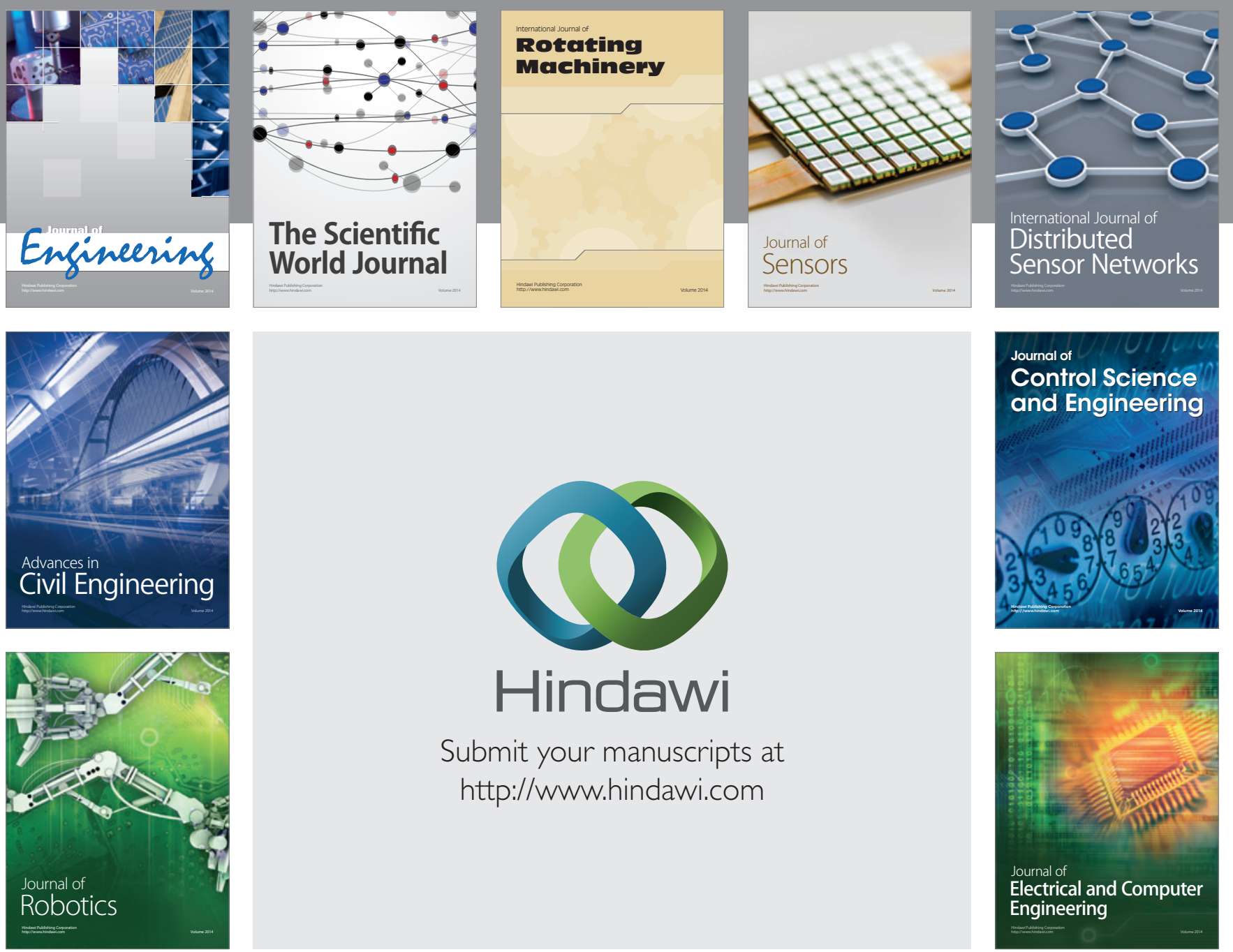

Submit your manuscripts at

http://www.hindawi.com
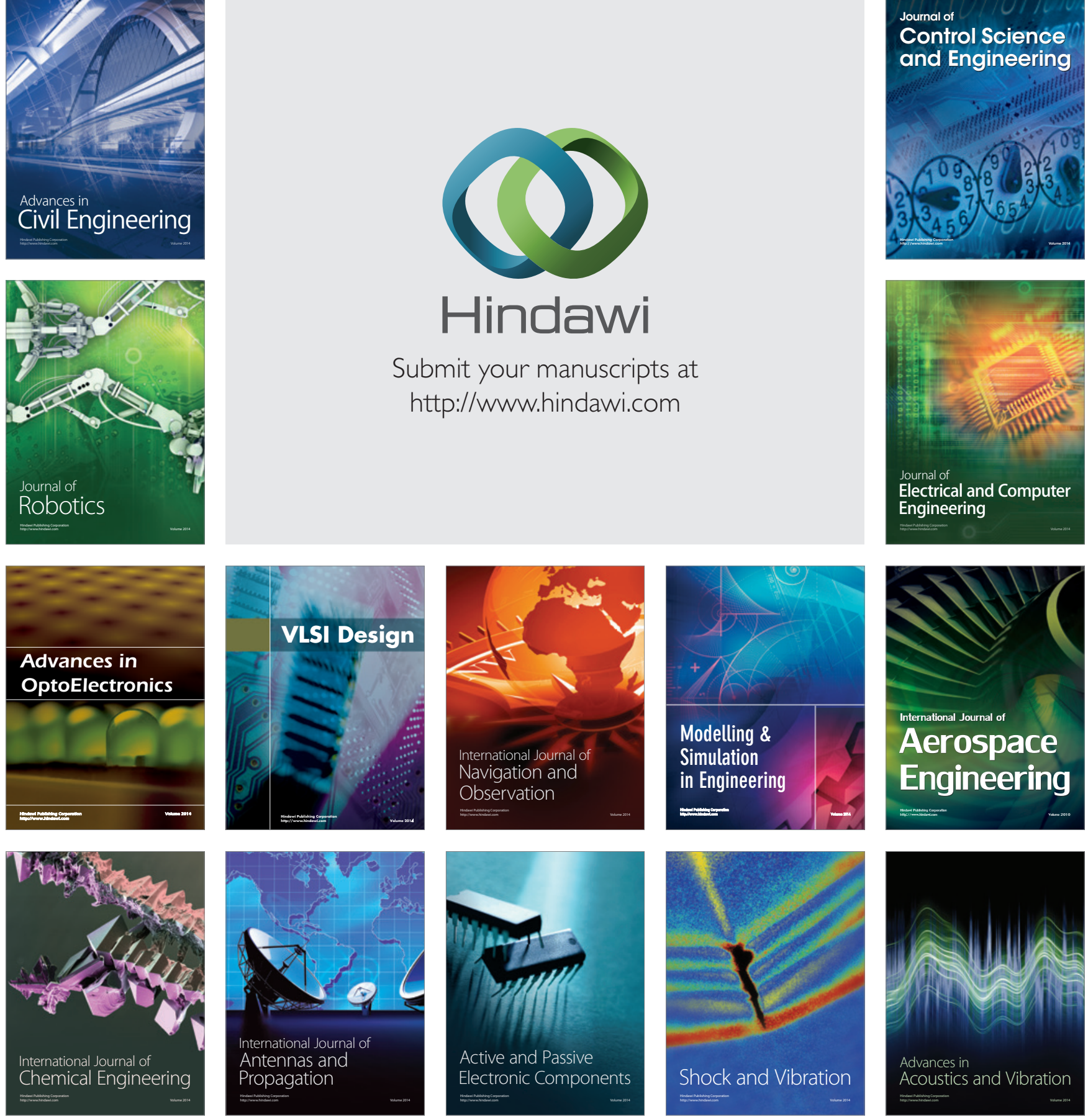